\title{
Constraining the Magnetic Field in the TeV Halo of Geminga with X-Ray Observations
}

\author{
Ruo-Yu Liu ${ }^{1}$ (D), Chong Ge ${ }^{2,3}$, Xiao-Na Sun ${ }^{4}$, and Xiang-Yu Wang ${ }^{4}$ \\ ${ }^{1}$ Deutsches Elektronen Synchrotron (DESY), Platanenallee 6, D-15738 Zeuthen, Germany \\ ${ }^{2}$ Purple Mountain Observatory, Chinese Academy of Sciences, Nanjing 210008, People's Republic of China \\ ${ }^{3}$ Department of Physics and Astronomy, University of Alabama in Huntsville, Huntsville, AL 35899, USA \\ ${ }^{4}$ School of Astronomy and Space Science, Nanjing University, Nanjing 210093, People's Republic of China \\ Received 2019 February 3; revised 2019 March 19; accepted 2019 March 20; published 2019 April 24
}

\begin{abstract}
Recently, the High Altitude Water Cherenkov (HAWC) collaboration reported the discovery of a TeV halo around the Geminga pulsar. The $\mathrm{TeV}$ emission is believed to originate from the inverse Compton scattering of pulsarinjected electrons/positrons off cosmic microwave background photons. During this time, these electrons should inevitably radiate X-ray photons via synchrotron radiation, providing a useful constraint on the magnetic field in the TeV halo. In this work, we analyze the data of XMM-Newton and Chandra, and obtain an upper limit for the diffuse X-ray flux in a $600^{\prime \prime}$ region around the Geminga pulsar, which is at a level of $\lesssim 10^{-14} \mathrm{erg} \mathrm{cm}^{-2} \mathrm{~s}^{-1}$. By numerically modeling both the $\mathrm{X}$-ray and $\mathrm{TeV}$ observations assuming the isotropic diffusion of injected electrons/ positrons, we find that the magnetic field inside the $\mathrm{TeV}$ halo is required to be $<1 \mu \mathrm{G}$, which is significantly weaker than the typical magnetic field in the interstellar medium. The weak magnetic field together with the small diffusion coefficient inferred from the HAWC observation implies that the Bohm limit of particle diffusion may probably have been achieved in the $\mathrm{TeV}$ halo. We also discuss alternative possibilities for the weak X-ray emission, such as the hadronic origin of the $\mathrm{TeV}$ emission or a specific magnetic field topology, in which a weak magnetic field and a very small diffusion coefficient might be avoided.
\end{abstract}

Key words: gamma rays: general - pulsars: individual (Geminga) - radiation mechanisms: non-thermal - X-rays: general

\section{Introduction}

Recently, the High Altitude Water Cherenkov (HAWC) collaboration reported the discovery of $\mathrm{TeV}$ gamma-ray halos around about $10^{\circ}$ of two nearby pulsars, Geminga and Monogem (Abeysekara et al. 2017). Given that pulsars are promising accelerators of cosmic-ray (CR) electrons/positrons (hereafter, for simplicity, we do not distinguish positrons from electrons unless specified), the extended $\mathrm{TeV}$ emissions probably arise from inverse Compton (IC) scattering of $\sim 10-100 \mathrm{TeV}$ electrons off cosmic microwave background photons. By jointly modeling the surface brightness profiles (SBPs) of these two $\mathrm{TeV}$ halos, the HAWC collaboration suggested a low diffusion coefficient of $D=(4.5 \pm 1.2) \times 10^{27} \mathrm{~cm}^{2} \mathrm{~s}^{-1}$ at $100 \mathrm{TeV}$ in the TeV halos.

The derived diffusion coefficient is more than two orders of magnitude smaller than the standard diffusion coefficient in the interstellar medium (ISM) that is inferred from the measurement of the secondary-to-primary ratio in the CR spectrum (i.e., the baron-to-carbon ratio; Aguilar et al. 2016). Hooper et al. (2017) pointed out that the electron spectrum measured by the H.E.S.S. extending to about $20 \mathrm{TeV}$ disfavors a small diffusion coefficient throughout the bulk of the local ISM. A plausible scenario, however, is the appearance of two diffusion zones between the pulsar and Earth, with the pulsar located inside an inefficient diffusion zone of size a few tens of parsecs, while the diffusion coefficient in the bulk of the ISM is the standard one inferred from the CR measurement. Such a scenario has been proposed in several previous works (Hooper \& Linden 2018; Fang et al. 2018; Profumo et al. 2018; Tang \& Piran 2019). However, the cause of the inefficient diffusion zone is still not clear. The inefficient diffusion zone may be the parent supernova remnant of the pulsar and the turbulence is driven by the shock (Fang et al. 2018), or it may be a relic pulsar wind nebula (PWN), the magnetic field of which is higher than that in the ISM and with a magnetic topology that could be complicated (Tang \& Piran 2019). Alternatively, the low diffusion coefficient could be caused by the instability, due to the gradient of CRs injected from the pulsar (Evoli et al. 2018; Quenby 2018).

For any turbulence-driven mechanism, the key is the growth of the plasma instability. The diffusion coefficient derived by the HAWC collaboration around the pulsars implies that the turbulence is nearly at saturation, with the perturbed magnetic field $\delta B$ comparable to the mean field $B$. Thus, the magnetic field in the $\mathrm{TeV}$ halo is very crucial in understanding the origin of the low diffusion coefficient. On the other hand, we note that the energy of electrons that radiate $\sim 20 \mathrm{TeV}$ photons via IC scattering off CMB photons is about $100 \mathrm{TeV}$. These electrons will inevitably radiate in the magnetic field via synchrotron radiation and most likely give rise to a diffuse $\mathrm{X}$-ray halo with a typical energy $\epsilon_{\mathrm{syn}} \sim 0.6\left(E_{e} / 100 \mathrm{TeV}\right)^{2}(B / 3 \mu \mathrm{G}) \mathrm{keV}$, with $E_{e}$ being the electron energy. The ratio between the diffuse $\mathrm{X}$-ray flux and the diffuse multi-TeV flux from the same region is approximately equal to the ratio between the magnetic field energy density in the $\mathrm{TeV}$ halo and the CMB energy density $U_{\mathrm{CMB}}$, i.e., $F_{\mathrm{keV}} / F_{10 \mathrm{TeV}} \simeq B^{2} / 8 \pi U_{\mathrm{CMB}}$. Thus, the diffuse $\mathrm{X}$-ray flux provides a clue to understand the magnetic field in the $\mathrm{TeV}$ halo.

In this work, we will study the magnetic field via the X-ray emission around Geminga. The remainder of this paper is organized as follows: in Section 2, we present our analysis of the X-ray data in the region around Geminga. In Section 3, we obtain the upper limit of the magnetic field in the $\mathrm{TeV}$ halo based on the theoretical modeling of the $\mathrm{TeV}$ and the $\mathrm{X}$-ray emission. In Section 4, we discuss the implications of our result and examine alternative interpretations. We give the conclusion in Section 5. 

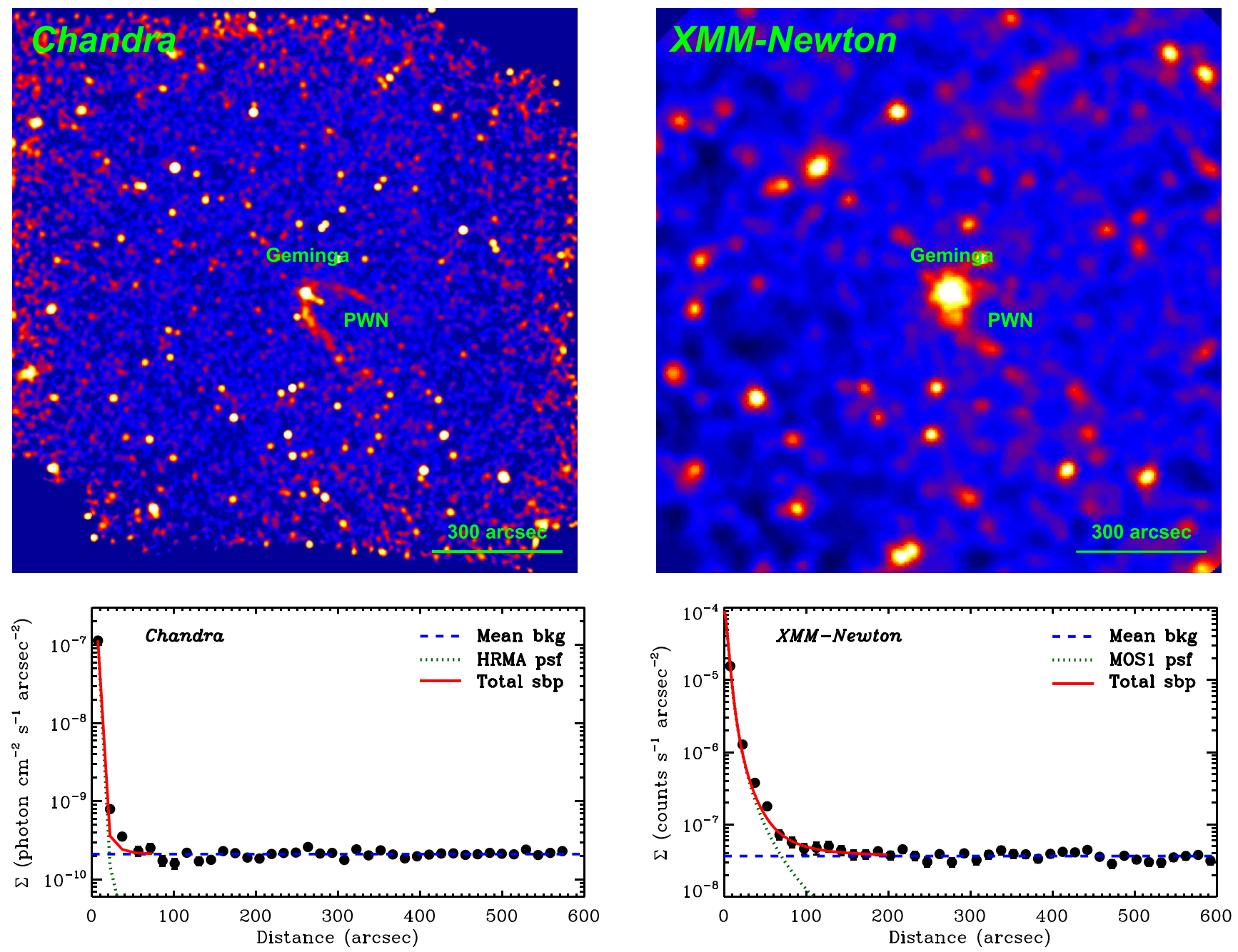

Figure 1. Upper panel: Chandra $0.7-2.0 \mathrm{keV}$ and XMM-Newton $0.7-1.3 \mathrm{keV}$ instrumental background-subtracted, exposure-corrected images. The central bright Geminga and its PWN are labeled. Lower panel: the diffuse SBP around Geminga. The central region of the SBP is dominated by the instrumental PSF, while the outer region of the SBP is flat. Thus, it is dominated by the cosmic background, shown with the blue dashed line.

Table 1

X-Ray Observations

\begin{tabular}{llccc}
\hline \hline Name & Chandra Data & Exposure (ks) & XMM-Newton Data & Exposure (ks) \\
\hline Geminga & 759214691146921469314694 & $659.3 / 660.7$ & 011117010102013501010311591001 & $187.4 / 191.1 / 292.2^{\mathrm{a}}$ \\
& 1555115552155951562215623 & & 040026020104002603010501270201 & 050127030105504102010550410301 \\
& 163181631916372 & & 0501270301 \\
\hline
\end{tabular}

Note.

${ }^{a}$ EPIC exposures of the clean MOS1/MOS2/total.

\section{Analysis of X-Ray Data}

Table 1 lists the Chandra and XMM-Newton observations used here. We reduce the X-ray data, following similar procedures to Ge et al. $(2018,2019)$ and briefly summarizing here, to study the diffuse X-ray emission around Geminga.

\subsection{Chandra}

We reduce the Chandra ACIS-I data with the Chandra Interactive Analysis of Observations (CIAO, version 4.9) and Calibration Database (CALDB, version 4.7.3). For each observation, we use the chandra_repro script with
VFAINT mode correction to reproduce a new level $=2$ event file. Then, we use deflare to filter the flares that deviate more than $3 \sigma$ from the mean count rate. The exposures of clean and original time are also included in Table 1. Point sources are detected by wavdetect. The point-spread function (PSF) of the Chandra High-Resolution Mirror Assembly (HRMA) shown in Figure 1 is modeled with the Chandra Ray Tracer (ChaRT) and simulate_psf. The instrumental stowed background is reprojected to match each of the ACIS-I chips, with (1) VFAINT cleaning, (2) point sources region masked, and (3) rescaled to the count rate in the $9.5-12 \mathrm{keV}$ band. We use merge_obs to produce the merged event and exposure 
maps from multiple observations. Then, the merged event image is subtracted with the merged stowed background image and further divided by the exposure map to get the flux image, from where we extract an SBP of the diffuse X-ray emission in $0.7-2.0 \mathrm{keV}$. Both the flux image and SBP are shown in Figure 1.

\subsection{XMM-Newton}

We process the XMM-Newton MOS data with the Extended Source Analysis Software (ESAS; Kuntz \& Snowden 2008; Snowden et al. 2008) as integrated into the XMM-Newton Science Analysis System (version 15.0.0) with the associated Current Calibration Files (CCF). The positive-negative (pn) data are in the small-window operating mode with a limited field of view (FOV); thus, we do not analyze the pn data. We reduce the raw event files from MOS with the emchain task. We use mos-filter to filter out the flares from solar soft protons. The MOS CCDs that are damaged or in an anomalous state are excluded in downstream processing. We apply the point source positions from Chandra to the XMM-Newton PSF from calview and Ghizzardi (2002), as well as the additional point sources detected by cheese in the outer radius, which is not covered by the Chandra observations. We use mosspectra to produce event images and exposure maps. The instrumental background images are modeled with mos_back. The residual soft-proton background images are modeled with protons. We combine the event images, background images, and exposure maps from multiple observations as well as from MOS1 and MOS2 with merge_comp_xmm. Then we produce a flux image and extract an SBP in $0.7-1.3 \mathrm{keV}$.

\subsection{The Diffuse X-Ray Emission}

The upper panel of Figure 1 shows the X-ray emission from Chandra and XMM-Newton. We then produce the SBPs after masking the point source (except the Geminga pulsar at the center) and the diffuse PWN around Geminga (e.g., Caraveo et al. 2003; Posselt et al. 2017). The diffuse X-ray SBP is shown in the lower panel of Figure 1. Excluding the central region affected by the instrument PSF, we do not find a flux drop toward larger radius like that in the $\mathrm{TeV}$ band measured by HAWC. Instead, the SBP is flat. We also compare the diffuse X-ray background within $10^{\circ}$ around Geminga using the RASS R45 (0.47-1.21 keV) flux. The fluxes from different radii are compatible with each other. Thus, the contribution of diffuse synchrotron radiation is not significant, and the diffuse $\mathrm{X}$-ray emission around Geminga is dominated by the cosmic background. We then estimate a $3 \sigma$ upper limit flux for the diffuse synchrotron radiation, assuming a power-law model, with the index $\Gamma=2.0$. The choice of power-law index does not affect our final estimate significantly given the narrow band that we consider. We ignore Galactic absorption because the Geminga is a quite nearby source $\left(d_{\text {pul }} \sim 250 \mathrm{pc}\right)$. The upper limit flux $\Phi_{n_{\sigma}}$ is estimated using Equation (3) in Hollowood et al. (2018),

$$
\Phi_{n_{\sigma}}=\Phi_{\text {model }} \cdot \frac{n_{\sigma} \sqrt{N_{\text {obs }}}}{N_{\text {model }}},
$$

where $\Phi_{\text {model }}$ is the model flux and $N_{\text {model }}$ is the product of the exposure time and the model count rate, $n_{\sigma}=3$ in our case, and $N_{\text {obs }}$ is the total observed number of cosmic background counts. The resultant $3 \sigma$ upper limit flux are $f_{\text {cha, } 0.7-2.0 \mathrm{keV}}=6.1 \times 10^{-15} \mathrm{erg} \mathrm{cm}^{-2} \mathrm{~s}^{-1}$ and $f_{\text {xmm }, 0.7-1.3 \mathrm{keV}}=5.0 \times 10^{-15} \mathrm{erg} \mathrm{cm}^{-2} \mathrm{~s}^{-1}$ within $600^{\prime \prime}$ from Chandra and XMM-Newton data, respectively.

\section{Constraint on the Magnetic Field in the TeV Halo}

The diffuse X-ray flux depends on the electron density and the strength of the magnetic field. Because the former quantity can be evaluated by modeling the HAWC observation, we first need to find out the electron distribution in the $\mathrm{TeV}$ halo and fit the observed multi-TeV flux and SBP measured by HAWC.

\subsection{Theoretical Modeling}

The Geminga pulsar has a high proper motion velocity of $211\left(d_{\text {pul }} / 250 \mathrm{pc}\right) \mathrm{km} \mathrm{s}^{-1}$ (Faherty et al. 2007), implying that the pulsar has moved $\sim 70 \mathrm{pc}$ from its birthplace given an age of $\tau_{\text {pul }}=342 \mathrm{kyr}$. However, the cooling timescale of $100 \mathrm{TeV}$ electrons, which is relevant for $\mathrm{TeV}$ and $\mathrm{X}$-ray emission, is several times $10^{11} \mathrm{~s}$ in ISM. For the best-fit diffusion coefficient obtained in the HAWC paper $\left(4.5 \times 10^{27} \mathrm{~cm}^{2} \mathrm{~s}^{-1}\right.$ at $100 \mathrm{TeV}), \mathrm{TeV}$ - and X-ray-emitting electrons diffuse a distance of $\sim 40 \mathrm{pc}$ while the pulsar only moves $\sim 5 \mathrm{pc}$ within the lifetime of these energetic electrons. We therefore ignore the proper motion of Geminga in this work for simplicity, and deal with the electron transport in the spherical coordinate, defining the pulsar location (i.e., particle injection) at $r=0$ and assuming spherical symmetry for the particle transport.

The differential density of electron with energy $E_{e}$ at a distance $r$ and at a time $t$ after the initial injection, i.e., $N\left(E_{e}, r, t\right)$, can be given by

$$
\begin{aligned}
\frac{\partial N\left(E_{e}, r, t\right)}{\partial t}= & \frac{1}{r^{2}} \frac{\partial}{\partial r}\left(r^{2} D\left(E_{e}, r\right) \frac{\partial N}{\partial r}\right) \\
& -\frac{\partial}{\partial E_{e}}\left(\dot{E}_{e} N\right)+Q\left(E_{e}, t\right) \delta(r),
\end{aligned}
$$

where $\delta(r)$ is the Dirac function. $D\left(E_{e}, r\right)$ is the diffusion coefficient at a distance $r$ from the pulsar, which is assumed to be

$$
D= \begin{cases}D_{1}, & r \leqslant r_{0} \\ D_{2}, & r>r_{0}\end{cases}
$$

with $r_{0}$ being the radius of the boundary between the inner inefficient diffusion region and the outer normal diffusion region. $\dot{E}_{e}$ is the cooling rate of electrons due to synchrotron radiation and IC radiation, which is given by Moderski et al. (2005) as

$$
\dot{E}_{e}=-\frac{4}{3} \sigma_{T} c\left(\frac{E_{e}}{m_{e} c^{2}}\right)^{2}\left[U_{B}+U_{\mathrm{ph}}\left(1+4 \frac{E_{e} \epsilon_{0}}{m_{e}^{2} c^{4}}\right)^{3 / 2}\right]
$$

where $\sigma_{\mathrm{T}}$ is the Thomson cross section, $m_{e}$ is the electron mass, and $c$ is the speed of light. $U_{B}=B^{2} / 8 \pi$ is the magnetic field energy density with $B$ following the same form as the diffusion coefficient, i.e.,

$$
B= \begin{cases}B_{1}, & r \leqslant r_{0} \\ B_{2}, & r>r_{0}\end{cases}
$$


and $U_{\mathrm{ph}}$ is the radiation field energy density. $\epsilon_{0}$ is the average photon energy of the radiation field, which is equal to $2.82 \mathrm{kT}$ in the case of blackbody or graybody radiation with $k$ being the Boltzmann constant and $T$ being the temperature. Following Abeysekara et al. (2017), in addition to the CMB, we also consider a $20 \mathrm{~K}$ infrared photon field and a $5000 \mathrm{~K}$ optical photon field as the background photon field. The spectra of both photon fields are assumed to follow a graybody distribution with an energy density of $0.3 \mathrm{eV} \mathrm{cm}^{-3}$, as approximately derived by GALPROP (Moskalenko \& Strong 1998). The injection spectrum of electrons at any given time $t$ is assumed to be in the form of a power-law function with a high-energy cutoff, i.e.,

$$
Q\left(E_{e}, t\right)=S(t) N_{0} E_{e}^{-p} e^{-E_{e} / E_{e, \max }},
$$

with $p$ being the spectral index and $E_{e, \max }$ being the maximum energy of the electrons injected by the pulsar, and $S(t) \propto 1 /\left(1+t / \tau_{s}\right)^{2}$, assuming the pulsar is a pure dipole radiator with a braking index of 3 , where $\tau_{s}$ is the spindown timescale of the pulsar and $\tau_{s}=12 \mathrm{kyr}$ is adopted. $N_{0}$ is the normalization and can be found from $\iint d t d E_{e} Q\left(E_{e}, t\right)=W_{e}$.

In order not to introduce too many free parameters, we fix $D_{2}=D_{\mathrm{ISM}}=3.86 \times 10^{28}\left(E_{e} / 1 \mathrm{GeV}\right)^{1 / 3} \mathrm{~cm}^{2} \mathrm{~s}^{-1}$ and $B_{2}=$ $3 \mu \mathrm{G}$ in our calculation. The energy dependence of $D_{1}$ is also assumed to be $\propto E^{1 / 3}$, following a Kolmogorov-type turbulence, i.e., $D_{1}=D_{1}(100 \mathrm{TeV})\left(E_{e} / 100 \mathrm{TeV}\right)^{1 / 3}$, while the normalization, i.e., $D_{1}(100 \mathrm{TeV})$, is allowed to change. On the other hand, we also require the value of $D_{1}$ at $E_{e, \max }$ to be larger than the Bohm diffusion coefficient $\left(D_{B}\left(E_{e, \max }\right)=r_{g}\left(E_{e, \max }\right) c / 3\right.$, where $r_{g}\left(E_{e, \max }\right)=E_{e, \max } / e B$ is the Larmor radius of the maximum-energy electron), which is the limiting case for the diffusion coefficient, so $D_{1}$ is not a totally free parameter. Because Xi et al. (2018) showed that a hard injection spectrum is required to be consistent with the upper limit of multi-GeV flux from the observation of Fermi-LAT, we fix $p=1.6$ for the moment. The spectral index is actually not important in this work. As we mentioned before, the energies of X-rayemitting electrons and $\mathrm{TeV}$-emitting electrons are quite close, so the expected X-ray flux is insensitive to the spectral index $p$ as long as the HAWC observation is fitted (see Section 3.3). Note that the photon index of the $\mathrm{TeV}$ emission measured by HAWC is -2.34 . Given a hard electron spectrum $p=1.6$, we need a relatively small cutoff in the injection electron spectrum to reproduce the observed spectrum, and therefore $E_{e, \max }=100-200 \mathrm{TeV}$ is employed. Such a high energy in principle can be achieved in the pulsar wind termination shock although some details on the acceleration mechanism remain unclear (see Kirk et al. 2009; Aharonian et al. 2013, and references therein). The minimum electron energy at injection is fixed at $1 \mathrm{GeV}$. Then, the free parameters left in our calculation are the total injection energy of electrons $W_{e}$, the magnetic field in the TeV halo $B_{1}$, and the halo diffusion coefficient $D_{1}(100 \mathrm{TeV})$.

We then solve the equation numerically, from $t=0$ when the pulsar just starts to inject electrons to the current age of the pulsar $\tau_{\text {pul }}$, by discretizing the equation into second-order accuracy in both space and energy dimensions, based on the method introduced in the Appendix. After obtaining the electron distribution, we can calculate the SBP from the radiation of the electrons. We focus on the contribution of electrons within a

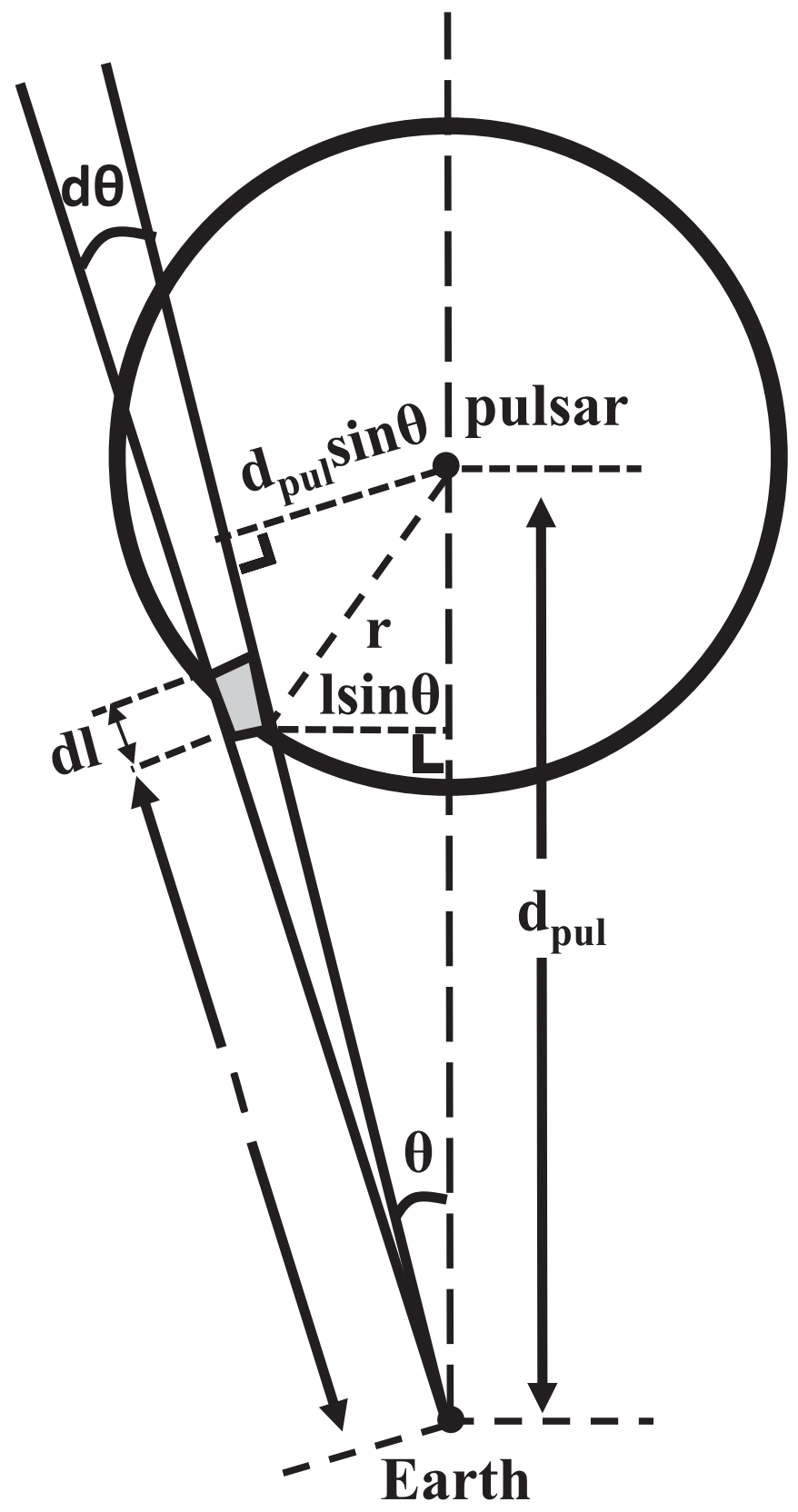

Figure 2. Diagrammatic sketch for calculating the integrated flux from the $\mathrm{TeV}$ halo. The system is symmetric with respect to the axis connecting Earth and the pulsar. The shaded region is the element volume in the integration. See Section 3.1 for details.

sphere of sufficiently large radius $r_{\max }{ }^{5}$ For a certain viewing angle $\theta$ from the pulsar's position, we integrate the emission of electrons in the line of sight, say, from a minimum distance of $l_{\text {min }}=d_{\text {pul }} \cos \theta-\sqrt{r_{\text {max }}^{2}-d_{\text {pul }}^{2} \sin ^{2} \theta}$ to Earth to a maximum distance of $l_{\text {max }}=d_{\mathrm{pul}} \cos \theta+\sqrt{r_{\max }^{2}-d_{\mathrm{pul}}^{2} \sin ^{2} \theta}$ to Earth (see Figure 2). At a given point in the line of sight with a distance $l$ to Earth $\left(l_{\min } \leqslant l \leqslant l_{\max }\right)$, the radius of this point from the pulsar $r$ can be found from $r=$ $\sqrt{l^{2}+d_{\mathrm{pul}}^{2}-2 l d_{\mathrm{pul}} \cos \theta}$, where $d_{\mathrm{pul}}$ is the distance between the pulsar and Earth, and the electron density can be obtained

\footnotetext{
5 We have tried the calculation with $r_{\max }=150,200,249$, and $249.99 \mathrm{pc}$; the results are almost the same.
} 
via interpolation based on the obtained $N\left(E_{e}, r, \tau_{\text {pul }}\right)$, where $\tau_{\text {pul }}$ is the age of the pulsar at the present time. Thus, the element volume in the neighborhood of this point can be given by $d V=2 \pi l \sin \theta \cdot l d \theta \cdot d l$, where $2 \pi$ takes into account the symmetry of the system. Let us define an operator $\mathcal{F}$, which calculates the differential spectrum of synchrotron radiation and IC radiation of electrons $F(\epsilon)$, following the formulae in Rybicki \& Lightman (1979), given the electron density, the magnetic field, or the background photon field. The flux emitted by electrons in the element volume can then be obtained from

$$
d F(\epsilon)=\mathcal{F}\left\{N\left[E_{e}, r(l), \tau_{\mathrm{pul}}\right], B[r(l)], T_{\mathrm{ph}}, U_{\mathrm{ph}}\right\} d V / 4 \pi l^{2} .
$$

Thus, the total photon flux from an annular region between $\theta$ and $\theta+d \theta$ centered at the pulsar in the celestial sphere can be given as

$$
\begin{aligned}
I(\epsilon, \theta) \cdot 2 \pi \sin \theta d \theta= & \int_{l_{\min }}^{l_{\max }} \mathcal{F}\left\{N\left[E_{e}, r(l), \tau_{\mathrm{pul}}\right],\right. \\
& \left.B[r(l)], T_{\mathrm{ph}}, U_{\mathrm{ph}}\right\} d V / 4 \pi l^{2},
\end{aligned}
$$

where $I(\epsilon, \theta)$ is the intensity of the annular region. Finally, we arrive at

$$
I(\epsilon, \theta)=\frac{1}{4 \pi} \int_{l_{\min }}^{l_{\max }} d l \mathcal{F}\left\{N\left[E_{e}, r(l), \tau_{\mathrm{pul}}\right], B[r(l)], T_{\mathrm{ph}}, U_{\mathrm{ph}}\right\}
$$

The total flux within a certain angle $\theta_{0}$ from the pulsar can be obtained from $F(\epsilon)=2 \pi \int_{0}^{\theta_{0}} I(\epsilon, \theta) \sin \theta d \theta$.

\subsection{Results}

We now apply the method to the TeV halos of Geminga $\left(d_{\mathrm{Gem}}=250 \mathrm{pc}, \tau_{\mathrm{Gem}}=342 \mathrm{kyr}\right)$. First, as an example, we show in the upper panel of Figure 3 the multiwavelength flux from a region within $10^{\circ}$ of Geminga (the black solid curve) and within $600^{\prime \prime}$ of Geminga (the blue solid curve) in the celestial sphere. The magnetic field and diffusion coefficient for the $\mathrm{TeV}$ halo are the same with best-fit parameters obtained in Abeysekara et al. (2017, i.e., $B_{1}=3 \mu \mathrm{G}$ and $D_{1}(100 \mathrm{TeV})=$ $4.5 \times 10^{27} \mathrm{~cm}^{2} \mathrm{~s}^{-1}$ ), and other parameters can be found in the caption of Figure 3. The theoretical SBP (the black solid curve) is compared to the observed one in the lower panel. As is shown, while the HAWC observation is explained, the associated X-ray flux overshoots the upper limits of XMMNewton and Chandra ${ }^{6}$ by one order of magnitude.

We find that $B_{1}$ needs to be tuned down to $\sim 0.8 \mu \mathrm{G}$ or smaller to be consistent with the upper limit of the X-ray flux. For such a small magnetic field, the cooling becomes inefficient. The cooling timescale of electrons $t_{c}=-E_{e} / \dot{E}_{e}$ becomes longer than that in the case of $B_{1}=3 \mu \mathrm{G}$, allowing $100 \mathrm{TeV}$ electrons to be transported to a farther distance before being cooled. Consequently, the SBP of the TeV emission would become too flat compared to the observed one. To make the SBP profile as steep as the observation, a smaller diffusion coefficient is required to keep more electrons closer to the pulsar, by making $\sqrt{D_{1} t_{c}}$ roughly remain the same as that in the reference case. For $100 \mathrm{TeV}$ electrons, the cooling time with

\footnotetext{
6 We only show the XMM-Newton upper limit in the figure for clarity, as the upper limits of these two instruments are basically the same.
}
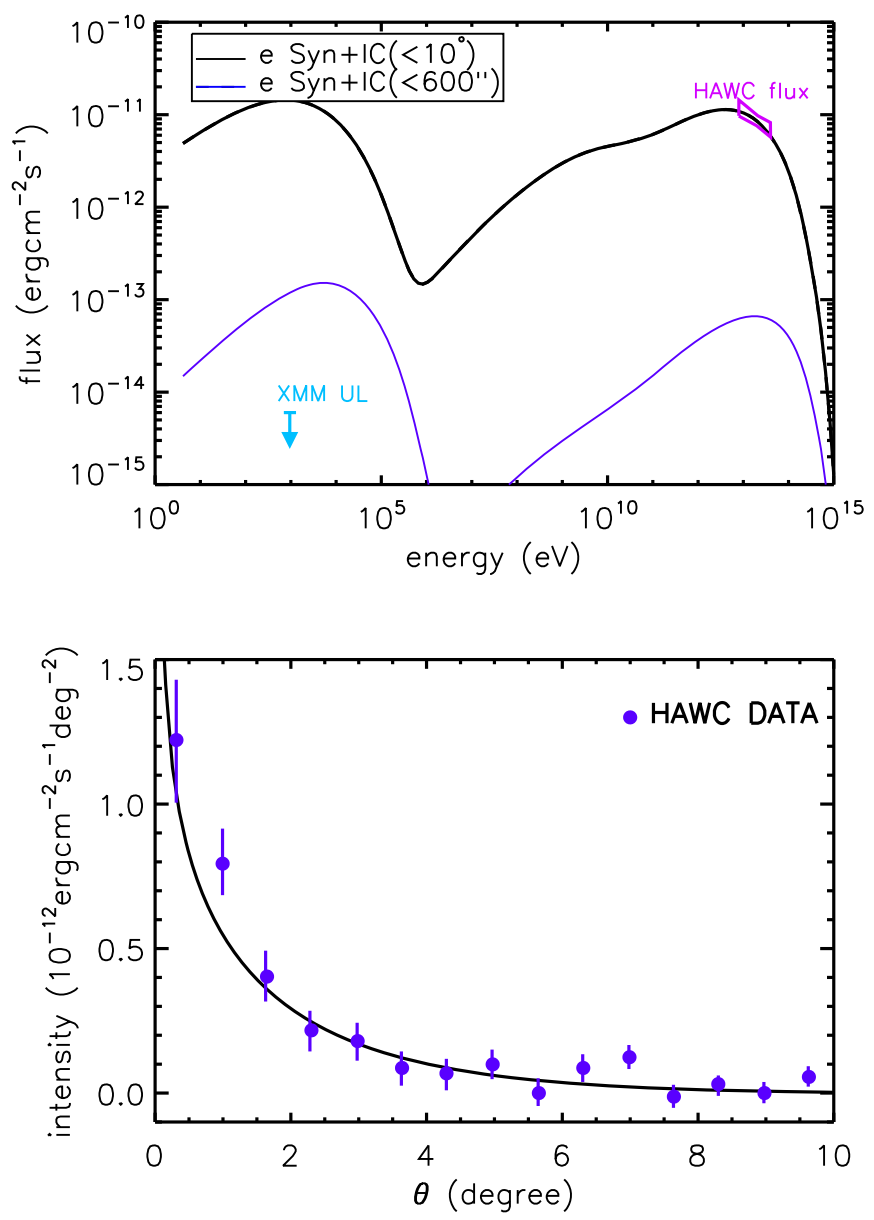

Figure 3. Upper panel: expected multiwavelength flux from the $\mathrm{TeV}$ halo of Geminga produced by the synchrotron and IC radiation of injected electrons, in comparison with data from various wavelengths. The black solid curve represents the expected flux from a region of $10^{\circ}$ around Geminga. The magenta bow tie shows the flux measured by HAWC from the same region (Abeysekara et al. 2017). The blue solid curve represents the integrated flux from a $600^{\prime \prime}$ region around Geminga, and the cyan arrow is the upper limit of XMM-Newton for the same region obtained in this work. The upper limit of Chandra almost overlaps with the $X M M$-Newton upper limit so we do not show it in the figure for clarity. Lower panel: the corresponding SBP of $8-40 \mathrm{TeV}$ emission. Employed parameters: $B_{1}=3 \mu \mathrm{G}, D_{1}(100 \mathrm{TeV})=4.5 \times 10^{27} \mathrm{~cm}^{2} \mathrm{~s}^{-1}, W_{e}=4.2 \times 10^{47} \mathrm{erg}, r_{0}=$ $50 \mathrm{pc}, E_{e, \max }=200 \mathrm{TeV}$, and $p=1.6$.

$B_{1}=3 \mu \mathrm{G}$ is $t_{c}=3 \times 10^{11} \mathrm{~s}$, while $t_{c}=9 \times 10^{11} \mathrm{~s}$ for $B_{1}=0.8 \mu \mathrm{G}$ (note that in this case the IC process dominates the cooling). Thus, it requires $D_{1}(100 \mathrm{TeV}) \simeq 1.6 \times$ $10^{27} \mathrm{~cm}^{2} \mathrm{~s}^{-1}$, which is, however, smaller than the Bohm diffusion coefficient for $B_{1}=0.8 \mu \mathrm{G}$ above a few tens of $\mathrm{TeV}$, i.e., $D_{B}=4.2 \times 10^{27}\left(E_{e} / 100 \mathrm{TeV}\right) \mathrm{cm}^{2} \mathrm{~s}^{-1}$. We try to fit the SBP with a physically reasonable diffusion coefficient by normalizing $D_{1}(100 \mathrm{TeV})$ to $D_{B}(100 \mathrm{TeV})$. The results are shown in the bottom-left panel of Figure 4, and we can see that the SBP is too flat compared to the observation (i.e., the intensity at a small angle is lower than the measured one). In the following, we denote this case as reference case A.

López-Coto \& Giacinti (2018) argued that a too weak magnetic field $(B<2 \mu \mathrm{G})$ can be ruled out according to the bad fitting to the SBP. However, if we relax the condition for a spatially constant diffusion coefficient inside the $\mathrm{TeV}$ halo, the fitting can be improved by considering a continuously decreasing diffusion coefficient with $r$ in the $\mathrm{TeV}$ halo within 

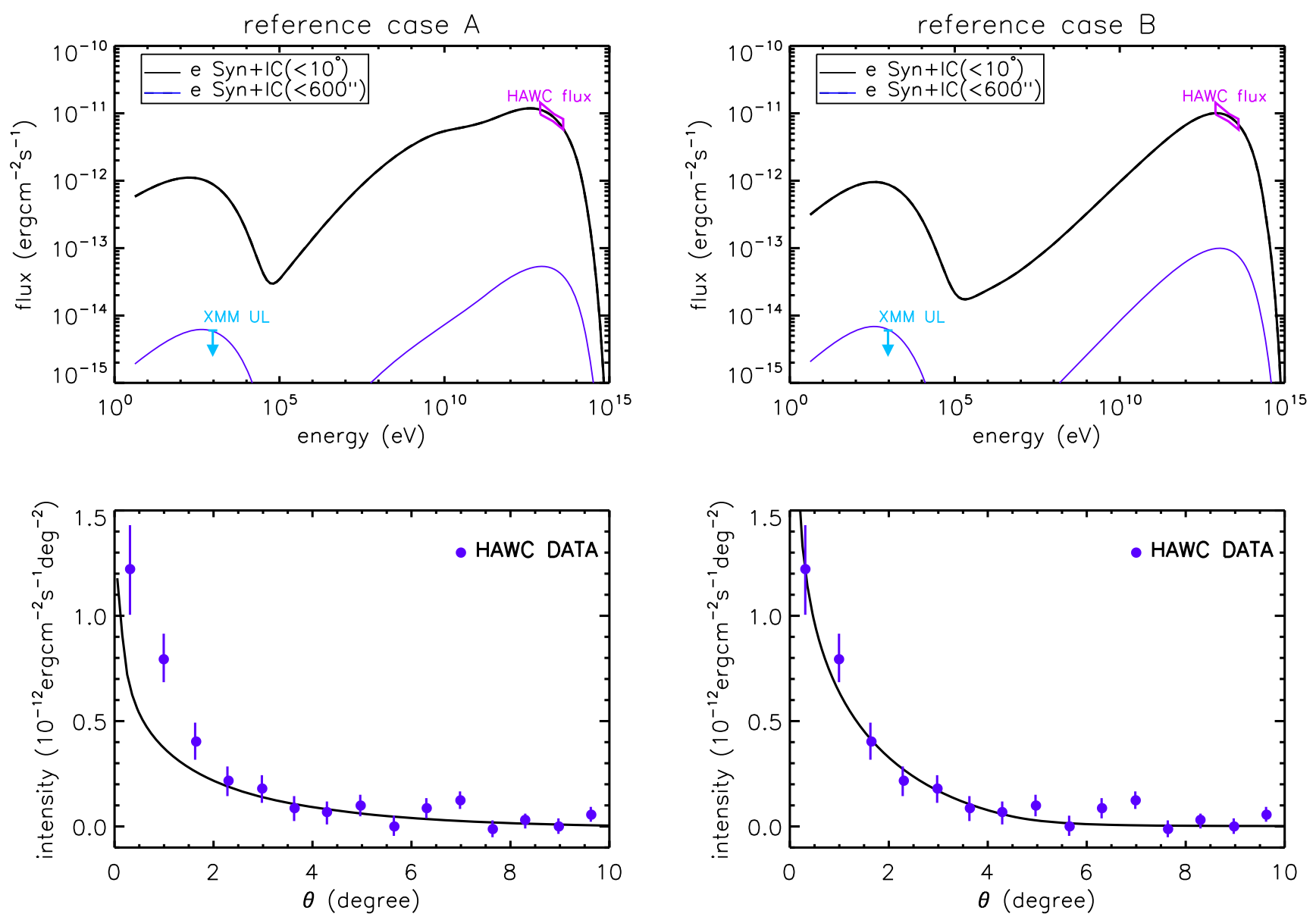

Figure 4. Same as Figure 3, but for a smaller magnetic field in the vicinity of Geminga $\left(B_{1}\right)$. Left: (reference case A) results under two diffusion zones with $B_{1}=0.8 \mu \mathrm{G}, D_{1}(100 \mathrm{TeV})=D_{B}(100 \mathrm{TeV})=4.2 \times 10^{27} \mathrm{~cm}^{2} \mathrm{~s}^{-1}, W_{e}=2.9 \times 10^{47} \mathrm{erg}$. Right: (reference case B) results under a continuously changing diffusion coefficient with $B_{1}=0.6 \mu \mathrm{G}, D_{1}(100 \mathrm{TeV})=D_{B}(100 \mathrm{TeV})=5.6 \times 10^{27} \mathrm{~cm}^{2} \mathrm{~s}^{-1}, W_{e}=7.7 \times 10^{47} \mathrm{erg}$. In both left and right panels, the maximum electron energy is fixed at $100 \mathrm{TeV}$.

$r_{0}$. Such a kind of diffusion coefficient might be possible, given that the CR self-confinement will be less efficient as the ionneutral damping of the generated waves can be more important at larger distance to the pulsar (e.g., Evoli et al. 2018). In this case, electrons diffuse faster as they propagate to larger radius, and hence the electron distribution will show a larger negative gradient outwards. Phenomenologically, there are multiple ways of constituting a function for $D(r)$ to give a consistent result with the observed morphology. As an example, we find the following form for $D$, i.e.,

$$
D=\left\{\begin{array}{l}
D_{1}, \quad r<20 \mathrm{pc} \\
D_{1}\left(\frac{D_{2}}{D_{1}}\right)^{(r-20) / 30}, \quad 20 \mathrm{pc} \leqslant r<50 \mathrm{pc}, \\
D_{2}=D_{\mathrm{ISM}}, \quad r \geqslant 50 \mathrm{pc}
\end{array}\right.
$$

with $D_{1}=5.6 \times 10^{27}\left(E_{e} / 100 \mathrm{TeV}\right)^{1 / 3} \mathrm{~cm}^{2} \mathrm{~s}^{-1}$ and $B_{1}=0.6 \mu \mathrm{G}$ giving a reasonable fitting to the data. Here, $5.6 \times 10^{27} \mathrm{~cm}^{2} \mathrm{~s}^{-1}$ is the Bohm diffusion coefficient at $100 \mathrm{TeV}$ under a magnetic field of $0.6 \mu \mathrm{G}$. The logarithm of the diffusion coefficient decreases linearly with $r$ from $D_{1}$ at $r=20 \mathrm{pc}$ to $D_{2}$ at $r=50 \mathrm{pc}$. The results are shown in the right panels of Figure 4. Note that an even smaller magnetic field is required inside the $\mathrm{TeV}$ halo to be consistent with the X-ray upper limit because of the steeper gradient of the electron density distribution obtained in this case. As a consequence, when the flux of $\mathrm{TeV}$ emission is fitted, there are more electrons distributed at a small radius than in the reference case $\mathrm{A}$, leading to a higher X-ray flux at the small radius. A weaker magnetic field $B_{1}$ is thus needed to reduce the expected X-ray flux. We denote this case as reference case B.

\subsection{Influence of Some Model Parameters}

In this section, we discuss the influence of some model parameters and demonstrate that the requirement of a weak magnetic field in the TeV halo of Geminga is robust.

First, we discuss the influence of $r_{0}$, i.e., the radius of the boundary between the inner inefficient diffusion zone and the outer standard diffusion zone. The HAWC measurement of the SBP suggests an inefficient diffusion zone extending to at least $\sim 30 \mathrm{pc}$ away from the Geminga pulsar. Thus, one can in principle assume $r_{0}$ to be any value $>30 \mathrm{pc}$. In the reference cases, we fix $r_{0}$ to be $50 \mathrm{pc}$, and a typical ISM magnetic field $B_{2}=B_{\text {ISM }}=3 \mu \mathrm{G}$ is assumed for $r>r_{0}$. A smaller $r_{0}$ cannot help avoid a weak magnetic field in the vicinity of the pulsar, because a steeper gradient of the electron distribution will be obtained for a smaller $r_{0}$. It will only result in a stronger X-ray flux from the small radius where the X-ray upper limit is extracted from so that an even weaker magnetic field has to be assumed for $r<r_{0}$. This is also the reason why a weaker magnetic field is required in reference case $B$ than in reference 

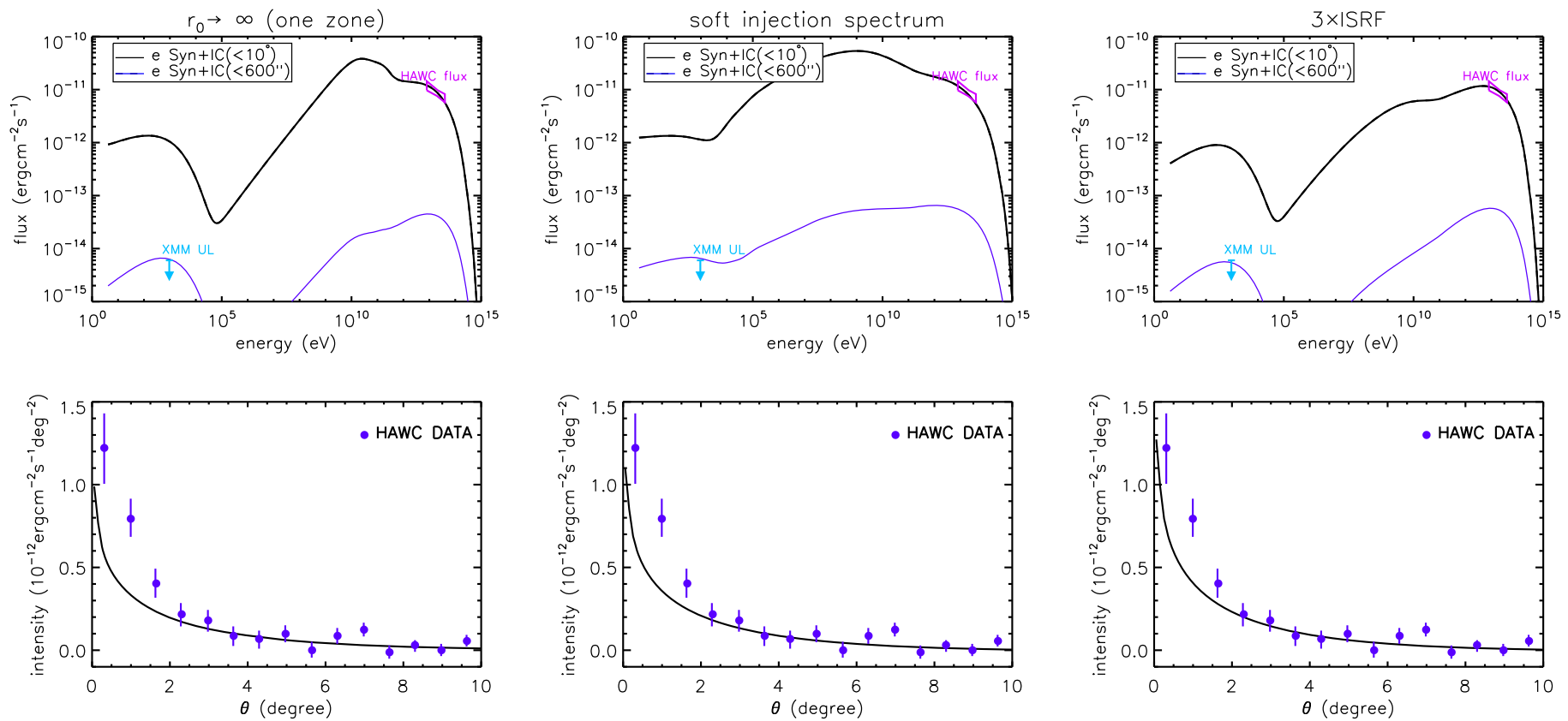

Figure 5. Influence of model parameters. Left: the case of $r_{0} \rightarrow \infty$ (one zone). $B_{1}=0.9 \mu \mathrm{G}, W_{e}=2.7 \times 10^{47} \mathrm{erg}$; Middle: the case of a soft injection spectrum for electrons with $p=2.2 . B_{1}=0.9 \mu \mathrm{G}, E_{e, \max }=200 \mathrm{TeV}, W_{e}=3.3 \times 10^{48} \mathrm{erg}$. Right: the case of three times higher density for the interstellar radiation field; $B_{1}=0.9 \mu \mathrm{G}$ and $W_{e}=2.4 \times 10^{47} \mathrm{erg}$. For all three cases, unlisted parameters are the same as those in reference case A. See Section 3.3 for more discussions

case $\mathrm{A}$ as discussed above. On the other hand, for a larger $r_{0}$, the weak magnetic field region will occupy a larger volume and hence more electrons will radiate in the weak magnetic field $B_{1}$. We thus question ourselves whether the value of $B_{1}$ can be increased up to a certain extent in this case. We then consider the limiting case of $r_{0} \rightarrow \infty$, which can also be regarded as only one diffusion zone. Despite the Fermi-LAT upper limit and the flat SBP due to inefficient cooling and inefficient diffusion of electrons, the value of $B_{1}$ can be increased, however, only to $0.9 \mu \mathrm{G}$ (see left panels of Figure 5). This is because the contribution of electrons far from the pulsar is subdominant as the electron density is quite low at large $r$, and therefore assuming a weak magnetic field for a much larger region does not reduce the synchrotron flux significantly.

In both reference cases, we fix the spectral index for injection electrons at $p=1.6$ and argue that the result is not sensitive to the injection spectral index, because the X-rayemitting electrons and the $\mathrm{TeV}$-emitting electrons are the same and thus the amount of the X-ray-emitting electrons can be normalized by the HAWC observation. While this argument is generally true, more precisely, the average energy of X-rayemitting electrons is a little higher than that of the TeV-emitting electrons especially given a weak magnetic field. One may then wonder whether a soft injection spectrum could reduce the X-ray flux and increase $B_{1}$ up to a certain extent. We here consider an injection spectral index of $p=2.2$. Again, regardless of the Fermi-LAT upper limit and the flat SBP, we find that while the TeV flux is reproduced, a magnetic field of $B_{1} \leqslant 0.9 \mu \mathrm{G}$, a little higher compared to that in reference cases, is needed in this case (see middle panels of Figure 5); this is, however, still significantly smaller than the typical ISM magnetic field. Although an even softer injection spectrum $(p>2.2)$ can further allow a larger $B_{1}$, the resulting $\mathrm{TeV}$ spectrum will be too soft to be consistent with the HAWC observation. From this figure, we can also see that the energy dependence $\delta$ of the diffusion coefficient cannot significantly influence the result either, as the energy of X-ray-emitting electrons and the energy of $\mathrm{TeV}$-emitting electrons are very close.

IC radiation competes with synchrotron radiation. The ratio of the peak flux of the former radiation process to the latter one is roughly proportional to the ratio of the background photon density to the magnetic field density. Although there are uncertainties in the interstellar infrared photon field and optical photon field, these two background photon fields actually are not very relevant to the $\mathrm{X}$-ray-emitting and $\mathrm{TeV}$-emitting electrons, due to the Klein-Nishina effect. Nevertheless, we artificially increase the energy densities of the infrared photon field and the optical photon field by a factor of 3 to see the possible influence. As shown in the right panels of Figure 5, the magnetic field for the inefficient diffusion zone (i.e., $B_{1}$ ) can be increased slightly to about $0.9 \mu \mathrm{G}$ in this case.

\section{Discussion}

\subsection{Low Magnetic Field in the Vicinity of the Pulsar}

Mattana et al. (2009) found that the ratio between the gamma-ray (1-30 TeV) and the X-ray (2-10 keV) flux of TeV PWNe and PWN candidates detected by H.E.S.S. increases with the characteristic age of the parent pulsars. This discovery is consistent with our result here, given an age of $342 \mathrm{kyr}$ for Geminga. The authors explained this empirical relation as the different cooling times of X-ray-emitting electrons (cooled) versus $\mathrm{TeV}$ gamma-ray-emitting electrons (uncooled), which is, however, not applicable to the case of Geminga, due to two reasons. First, the obtained X-ray upper limit is in the range of $0.7-1.3 \mathrm{keV}$ and the gamma-ray emission measured by HAWC is in the range of $8-40 \mathrm{TeV}$; these arise from electrons with quite close energies via synchrotron radiation and IC radiation, respectively. What is more, even if the energy of gamma-ray radiating electrons is smaller than that of X-ray-emitting electrons, the Geminga pulsar is old enough for gamma-ray- 
emitting electrons to be cooled as well. Thus, the null detection of diffuse X-ray emission from the TeV halo results from a low magnetic field. Note that, although the X-ray observation only focuses on a very small region of a projected size of $\lesssim 1 \mathrm{pc}$, the measured flux is contributed by all of the electrons in the line of sight, and hence the size of the low magnetic field region is required to be at least a few tens of parsecs. The obtained upper limit for the magnetic field in the vicinity of Geminga is much weaker than the typical ISM magnetic field. This may imply that the PWN of Geminga has experienced a significant expansion, and its size is much larger than the nebula observed in X-rays, which is $<0.1 \mathrm{pc}$ (Caraveo et al. 2003). A relatively weak magnetic field is also inferred in another TeV PWN, HESS J1825-137 (Aharonian et al. 2006), with a size of a few tens of parsecs. Because the TeV luminosity of this PWN is higher than the current spindown luminosity of the parent pulsar, a natural explanation of the origin of the $\mathrm{TeV}$ nebula would then be the "relic" multi-TeV electrons injected in the past when the spindown power of the pulsar was much higher. The survival of the multi-TeV electrons requires the magnetic field not to significantly exceed $1 \mu \mathrm{G}$ (Aharonian et al. 2006, 2013).

The low diffusion coefficient resulting from the HAWC observation together with the low magnetic field suggests a highly turbulent magnetic field in the $\mathrm{TeV}$ halo with field perturbation $\delta B / B$ of unity. Plasma instabilities, such as the streaming instability driven by the $\mathrm{CR}$ gradient, must grow efficiently to saturation for wave number $k<2 \pi / r_{g}(100 \mathrm{TeV})$. Quenby (2018) and Evoli et al. (2018) suggested that CR selfregulation can be important around Geminga. A self-consistent study including the growth of the streaming instability, its feedback on the CR transport, as well as modeling the multiwavelength emission is needed to justify this possibility.

\subsection{A Hadronic Origin Scenario for the TeV Emission}

Because pulsars and PWNe have been suggested as accelerators of CR protons (e.g., Cheng et al. 1986; Gallant et al. 1999; Arons 2003; Lemoine et al. 2015), we now investigate whether such a weak magnetic field can be avoided if we ascribe the $\mathrm{TeV}$ halo to the $p p$ collision between the accelerated protons and the surrounding ISM, while the electron injection from the pulsar can be very low in this case. Owing to the inefficient cooling of protons, historically injected protons can survive to the present day. Because the spindown power of the pulsar was higher in the past, the accumulated protons might provide a sufficient energy budget.

The gas density in the very vicinity of Geminga is about $n_{g} \sim 0.1 \mathrm{~cm}^{-3}$, as inferred from the equilibrium between the ram pressure of the pulsar wind and the thermal pressure of the ISM (Caraveo et al. 2003). Such a low gas density results in a low energy-loss rate of protons via the $p p$ collision, i.e., $\frac{1}{E_{p}} \frac{d E_{p}}{d t} \simeq$ $0.17 \sigma_{p p} n_{g} c=2 \times 10^{-17} \mathrm{~s}^{-1}$. Given that the $8-40 \mathrm{TeV}$ luminosity within $10^{\circ}$ of Geminga is about $L_{\mathrm{TeV}}=10^{32} \mathrm{erg} \mathrm{s}^{-1}$, it requires the energy of $\sim 80-400 \mathrm{TeV}$ CR protons inside the $\mathrm{TeV}$ halo to be $L_{\mathrm{TeV}} /\left(\frac{1}{E_{p}} \frac{d E_{p}}{d t}\right)=5 \times 10^{48} \mathrm{erg}$. Such an amount of $\mathrm{CR}$ proton energy is only a fraction of the total spindown power of Geminga (which is a few times $10^{49} \mathrm{erg}$ ). On the other hand, a continuously decreasing diffusion coefficient with $r$ similar to that in reference case $\mathrm{B}$ is also needed in the hadronic
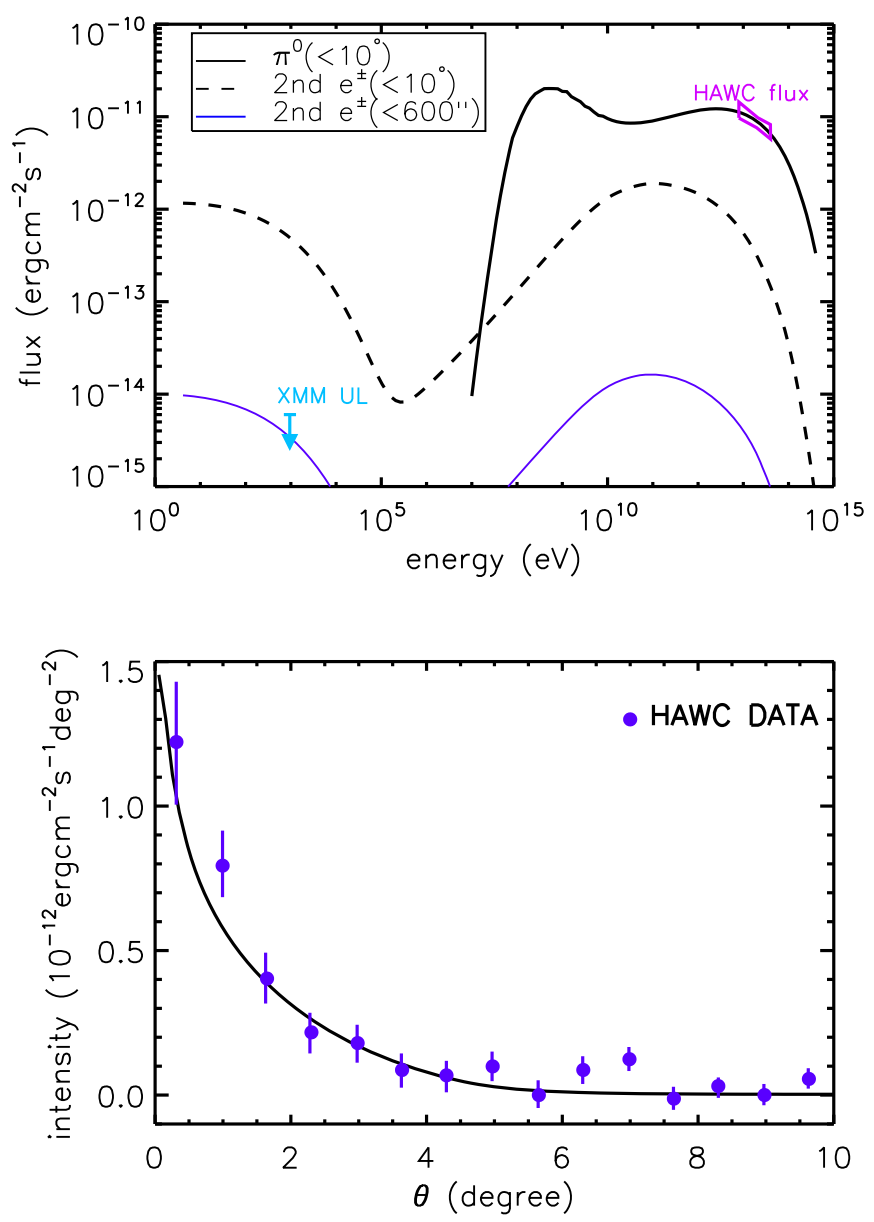

Figure 6. The expected multiwavelength flux (upper panel) and SBP in the hadronic model (lower panel). The black solid curve is the pionic emission arising from $p p$ collision. The synchrotron and IC emission from secondary electrons within $10^{\circ}$ of Geminga (black dashed curve) and within $600^{\prime \prime}$ of Geminga (blue curve) are also shown. The gas density is $n_{g}=0.1 \mathrm{~cm}^{-3}$ and the required total proton energy to fit the $\mathrm{TeV}$ flux is unreasonably high, i.e., $W_{p}=1.6 \times 10^{52} \mathrm{erg}$. See Section 4.2 for more discussion.

interpretation, as otherwise the expected SBP would be too flat to be consistent with the observed one given the inefficient cooling. However, lots of protons will then escape to much farther distances, and a huge amount of injection proton energy is consequently required.

In Figure 6, we show the expected multiwavelength flux and the SBP in the hadronic scenario, with $B_{1}=B_{2}=3 \mu \mathrm{G}$. The maximum injection proton energy is assumed to be $500 \mathrm{TeV}$. The diffusion coefficient follows the form of Equation (10) with $D_{1}=3.3 \times 10^{27}\left(E_{p} / 100 \mathrm{TeV}\right)^{1 / 3} \mathrm{~cm}^{2} \mathrm{~s}^{-1}$, through the normalization $D_{1}(500 \mathrm{TeV})=D_{B}(500 \mathrm{TeV})$ for $B_{1}=3 \mu \mathrm{G}$. With this diffusion coefficient, we find that the proton energy at $100 \mathrm{TeV}$ inside the inner diffusion zone is only $\sim 0.1 \%$ of total injected energy at $100 \mathrm{TeV}$. As a result, the required total proton injection energy is found to be $\sim 10^{52}\left(n_{g} / 0.1 \mathrm{~cm}^{-3}\right)^{-1} \mathrm{erg}$, which is much larger than the spindown energy of Geminga and the CR energy that a supernova remnant can supply, even for the typical ISM density of $n_{g}=1 \mathrm{~cm}^{-3}$. The proton injection energy can be reduced if more protons are injected at late times, such as with a constant injection luminosity (i.e., $S(t) \propto t^{0}$ ). However, the required proton injection energy in this case is still $\sim 10^{50}\left(n_{g} / 1 \mathrm{~cm}^{-3}\right)^{-1} \mathrm{erg}$. Moreover, taking into account the 
proper motion of Geminga will lead to a larger demand on the energy budget, as the proton distribution will be more extended. One may employ an even smaller diffusion coefficient (on the order of $10^{26} \mathrm{~cm}^{2} \mathrm{~s}^{-1}$ at $100 \mathrm{TeV}$ ) to further reduce the required proton energy. However, a larger magnetic field has to be invoked in this case to ensure that the employed diffusion coefficient is larger than that in the Bohm limit. Note that we do not consider electron injection in this case, but there are still secondary electrons produced in the $p p$ collisions accompanied by gamma rays. As is shown with the blue curve in the upper panel of Figure 6, the synchrotron radiation of secondary electrons is only marginally consistent with the X-ray upper limit with $B_{1}=3 \mu \mathrm{G}$. A larger magnetic field will result in a higher synchrotron flux of the secondary electrons and contradict the X-ray upper limit. Thus, we conclude that a simple hadronic model is not a preferable solution.

\subsection{A Scenario of an Ordered Magnetic Field inside the TeV Halo}

Before we make our conclusion, we would like to qualitatively discuss another possible scenario for the null detection of diffuse X-ray emission, in which the need for a weak magnetic field may be avoided. Let us denote the pitch angle between an electron and the magnetic field by $\alpha$. Due to the relativistic beaming effect, the synchrotron radiation of the electron with $E_{e}$ will be concentrated to a very small angle $1 /\left(E_{e} / m_{e} c^{2}\right)$ of a cone with half-opening angle $\alpha$. On the other hand, the synchrotron radiation power is $P_{\text {syn }} \propto(B \sin \alpha)^{2}$. Thus, if the magnetic field in the $\mathrm{TeV}$ halo of Geminga is roughly aligned with (or oppositely aligned with) our line of sight to the pulsar, the synchrotron radiation of electrons that move toward us will be very weak given $\sin ^{2} \alpha \ll 1$, while we cannot see the efficient synchrotron radiation of electrons with a large pitch angle because their radiation will be beamed into the other direction.

Note that the obtained results in previous sections are based on isotropic particle diffusion. However, electrons would experience anisotropic diffusion in the presence of such a mean magnetic field orientation in the TeV halo. Particles will diffuse faster in the direction parallel to the mean field than in the direction perpendicular to the mean field. The TeV SBP may still be explained as it reflects the projected electron distribution, which is mainly relevant for the perpendicular diffusion. Interestingly, the expected positron flux at Earth contributed by Geminga can be much higher than that expected in the isotropic diffusion scenario. Detailed modeling in this scenario will be helpful for verification. Such a calculation is not available with our current code, but will be an interesting project in the future.

\section{Conclusion}

In this work, we analyzed the data of XMM-Newton and Chandra in a $600^{\prime \prime}$ region around the Geminga pulsar. No significant X-ray emission is detected, yielding an upper limit of $(5-6) \times 10^{-15} \mathrm{erg} \mathrm{cm}^{-2} \mathrm{~s}^{-1}$ in $0.7-2.0 \mathrm{keV}$ for the diffuse $\mathrm{X}$-ray flux. We then modeled the $\mathrm{TeV}$ emission measured by HAWC under the constraint of the X-ray upper limit. By solving the 1D transport equation of injected electrons, we obtained the spatial distribution of electrons and then calculated the expected multiwavelength flux of electrons from the $\mathrm{TeV}$ halo via synchrotron radiation and IC radiation. On the premise of fitting the $\mathrm{TeV}$ emission, we found that the magnetic field in the $\mathrm{TeV}$ halo needs to be smaller than $0.8 \mu \mathrm{G}$ in order not to overshoot the X-ray upper limit. The low magnetic field may imply that the PWN of Geminga has experienced significant expansion to a size much larger than the $<0.1 \mathrm{pc}$ nebula observed in X-ray. The weak magnetic field together with the small diffusion coefficient inferred from the HAWC observation implies that the Bohm diffusion may probably have been achieved in the $\mathrm{TeV}$ halo. We also sought alternative explanations for the null detection of diffuse X-ray emission without invoking a weak magnetic field and/or a small diffusion coefficient. We found that the hadronic interpretation of Geminga's TeV halo does not work, because it requires extreme parameters such as a huge amount of proton injection energy and/or a very small diffusion coefficient. On the other hand, both the weak magnetic field and the small (parallel) diffusion coefficient may be avoided if the magnetic field in the $\mathrm{TeV}$ halo has a mean direction roughly (oppositely) aligned with our line of sight.

We thank Huirong Yan and Zhiyuan $\mathrm{Li}$ for helpful discussion. C.G. acknowledges support from the National Natural Science Foundation of China No. 11703090. X.-Y.W. is supported by the National Key R\&D program of China under grant No. 2018 YFA0404200 and NSFC grant Nos. 11625312 and 11851304.

\section{Appendix \\ Discretizing the Particle Transport Equation}

To solve Equation (2), we employ the operator-splitting technique to simplify the problem into solving a parabolic partial differential equation (the diffusion in space) and a hyperbolic partial differential equation (the convection in energy space). The classical Strang splitting scheme which is of second-order accuracy is adopted so that when going from the $l$ th to the $l+1$ th time step with the step length $\Delta t$, we have

$$
N^{l+1}\left(r, E_{e}\right)=N^{l}\left(r, E_{e}\right) e^{\mathcal{L}_{E} \Delta t / 2} e^{\mathcal{L}_{r} \Delta t} e^{\mathcal{L}_{E} \Delta t / 2},
$$

where $\mathcal{L}_{E}$ and $\mathcal{L}_{r}$ are the differential operators for energy $E_{e}$ and radius $r$, respectively.

The implicit second-order upwind scheme is employed for the discretization of the energy term, i.e.,

$$
\begin{aligned}
\frac{N_{i, j}^{l+1}-N_{i, j}^{l}}{\Delta t}= & \frac{1}{2}\left[\frac{-b_{i, j+2} N_{i, j+2}^{l+1}+4 b_{i, j+1} N_{i, j+1}^{l+1}-3 b_{i, j} N_{i, j}^{l+1}}{2 \Delta E}\right. \\
& \left.+\frac{-b_{i, j+2} N_{i, j+2}^{l}+4 b_{i, j+1} N_{i, j+1}^{l}-3 b_{i, j} N_{i, j}^{l}}{2 \Delta E}\right],
\end{aligned}
$$

where $i$ is the index of the spatial step and $j$ is the index of the energy step. After a few manipulations, we obtain

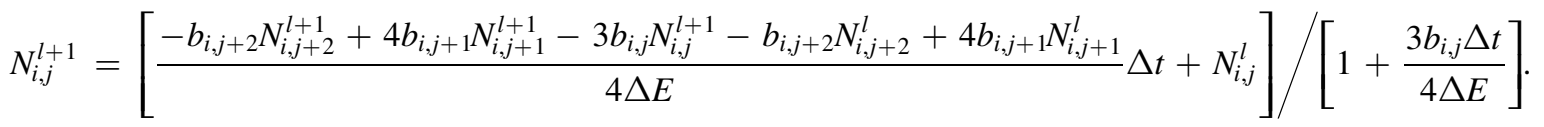


Given the boundary condition $N_{i, j_{\max }}=0$ for any $l$, we can solve $N_{i, j}^{l+1}$ from $j=j_{\max }-1$ to $j=0$.

For the discretization of the spatial term, we adopt the finite volume method of the implicit scheme, which leads to

$$
\begin{aligned}
\frac{N_{i, j}^{l+1}-N_{i, j}^{l}}{\Delta t}= & \frac{D_{i+1 / 2, j} r_{i+1 / 2}^{2}}{2 \Delta r^{2} r_{i}^{2}} \\
& \times\left(N_{i+1, j}^{l}-N_{i, j}^{l}+N_{i+1, j}^{l+1}-N_{i, j}^{l+1}\right) \\
& -\frac{D_{i-1 / 2, j} r_{i-1 / 2}^{2}}{2 \Delta r^{2} r_{i}^{2}}\left(N_{i, j}^{l}-N_{i-1, j}^{l}\right. \\
& \left.+N_{i, j}^{l+1}-N_{i-1, j}^{l+1}\right),
\end{aligned}
$$

where $r_{i+1 / 2}=r_{i}+\Delta r / 2, \quad r_{i-1 / 2}=r_{i}-\Delta r / 2, \quad D_{i+1 / 2, j}=$ $\left(D_{i, j}+D_{i+1, j}\right) / 2, \quad$ and $\quad D_{i-1 / 2, j}=\left(D_{i-1, j}+D_{i, j}\right) / 2$. This equation can be rearranged into

$$
\begin{aligned}
& -\frac{\left(D_{i, j}+D_{i-1, j}\right) \Delta t}{4 \Delta r^{2}}\left(1-\frac{\Delta r}{r_{i}}\right) N_{i-1, j}^{l+1} \\
& +\left[1+\frac{\left(D_{i, j}+D_{i+1, j}\right) \Delta t}{4 \Delta r^{2}}\left(1+\frac{\Delta r}{r_{i}}\right)\right. \\
& \left.+\frac{\left(D_{i, j}+D_{i-1, j}\right) \Delta t}{4 \Delta r^{2}}\left(1-\frac{\Delta r}{r_{i}}\right)\right] N_{i, j}^{l+1} \\
& -\frac{\left(D_{i, j}+D_{i+1, j}\right) \Delta t}{4 \Delta r^{2}}\left(1+\frac{\Delta r}{r_{i}}\right) N_{i+1, j}^{l+1} \\
& =\frac{\left(D_{i, j}+D_{i-1, j}\right) \Delta t}{4 \Delta r^{2}}\left(1-\frac{\Delta r}{r_{i}}\right) N_{i-1, j}^{l} \\
& +\left[1-\frac{\left(D_{i, j}+D_{i+1, j}\right) \Delta t}{4 \Delta r^{2}}\left(1+\frac{\Delta r}{r_{i}}\right)\right. \\
& \left.-\frac{\left(D_{i, j}+D_{i-1, j}\right) \Delta t}{4 \Delta r^{2}}\left(1-\frac{\Delta r}{r_{i}}\right)\right] N_{i, j}^{l} \\
& +\frac{\left(D_{i, j}+D_{i+1, j}\right) \Delta t}{4 \Delta r^{2}}\left(1+\frac{\Delta r}{r_{i}}\right) N_{i+1, j}^{l},
\end{aligned}
$$

which is a tridiagonal system and can be easily solved. Note that the above equation is for a continuous $D(r)$. If $D(r)$ follows the form of the step function as is shown in Equation (3), we have $D_{i-1 / 2, j}=D_{i, j}$ and $D_{i+1 / 2, j}=D_{i+1, j}$. Thus, all the terms of $\left(D_{i, j}+D_{i-1, j}\right)$ in the numerator need to be be replaced by $2 D_{i, j}$, and all the terms of $\left(D_{i, j}+D_{i+1, j}\right)$ need to be replaced by $2 D_{i+1, j}$ in Equation (15). For the outer boundary where $i=i_{\max }$ (corresponding to a sufficiently large distance to the pulsar), we impose $N_{i_{\max }, j}=0$ for any $l$. For the inner boundary where $i=0$ or $r_{1}=0$, we utilize the symmetry of the system, i.e., $\partial N / \partial r=0$, leading to $N_{-1, j}=N_{1, j}$ and $D_{-1, j}=D_{1, j}$.
Particles are assumed to inject from $r=0$, which is embodied by the Dirac function $\delta(r)$ in Equation (2). We adopt a rectangular function to approximate the Dirac function, i.e.,

$$
Q\left(E_{e}, t\right) \delta(r)=\frac{Q\left(E_{e}, t\right)}{4 \pi \Delta r^{3}} \times\left\{\begin{array}{ll}
1, & i=0,1 \\
0, & i>1
\end{array} .\right.
$$

In our calculation, we set $\Delta t=5 \mathrm{yr}, \Delta r=0.25 \mathrm{pc}$, with $r_{i_{\max }}=2.5 \mathrm{kpc}$. We divide the energy in logarithmic space with $\Delta \lg E=1 / 300$ between $E_{0}=0.1 \mathrm{GeV}$ and $E_{j_{\max }}=0.1 \mathrm{EeV}$.

\section{ORCID iDs}

Ruo-Yu Liu (i) https://orcid.org/0000-0003-1576-0961

\section{References}

Abeysekara, A. U., Albert, A., Alfaro, R., et al. 2017, Sci, 358, 911

Aguilar, M., Ali Cavasonza, L., Ambrosi, G., et al. 2016, PhRvL, 117, 231102

Aharonian, F., Akhperjanian, A. G., Bazer-Bachi, A. R., et al. 2006, A\&A, 460,365

Aharonian, F., Bergström, L., \& Dermer, C. 2013, Astrophysics at Very High Energies: Saas-Fee Advanced Course 40, Swiss Society for Astrophysics and Astronomy, Vol. 40 (Berlin: Springer), 40

Arons, J. 2003, ApJ, 589, 871

Caraveo, P. A., Bignami, G. F., De Luca, A., et al. 2003, Sci, 301, 1345

Cheng, K. S., Ho, C., \& Ruderman, M. 1986, ApJ, 300, 500

Evoli, C., Linden, T., \& Morlino, G. 2018, PhRvD, 98, 063017

Faherty, J., Walter, F. M., \& Anderson, J. 2007, Ap\&SS, 308, 225

Fang, K., Bi, X.-J., Yin, P.-F., \& Yuan, Q. 2018, ApJ, 863, 30

Gallant, Y. A., Achterberg, A., \& Kirk, J. G. 1999, A\&AS, 138, 549

Ge, C., Sun, M., Rozo, E., et al. 2019, MNRAS, 484, 1946

Ge, C., Wang, Q. D., Burchett, J. N., et al. 2018, MNRAS, 481, 4111

Ghizzardi, S. 2002, Flight Calibration of the PSF for the PN camera, Calibration document XMM-SOC-CAL-TN-0029, European Space Agency

Hollowood, D. L., Jeltema, T., Chen, X., et al. 2018, arXiv:1808.06637

Hooper, D., Cholis, I., Linden, T., \& Fang, K. 2017, PhRvD, 96, 103013

Hooper, D., \& Linden, T. 2018, PhRvD, 98, 083009

Kirk, J. G., Lyubarsky, Y., \& Petri, J. 2009, in Astrophysics and Space Science Library, Vol. 357, ed. W. Becker (Berlin: Springer), 421

Kuntz, K. D., \& Snowden, S. L. 2008, A\&A, 478, 575

Lemoine, M., Kotera, K., \& Pétri, J. 2015, JCAP, 7, 016

López-Coto, R., \& Giacinti, G. 2018, MNRAS, 479, 4526

Mattana, F., Falanga, M., Götz, D., et al. 2009, ApJ, 694, 12

Moderski, R., Sikora, M., Coppi, P. S., \& Aharonian, F. 2005, MNRAS, 363,954

Moskalenko, I. V., \& Strong, A. W. 1998, ApJ, 493, 694

Posselt, B., Pavlov, G. G., Slane, P. O., et al. 2017, ApJ, 835, 66

Profumo, S., Reynoso-Cordova, J., Kaaz, N., \& Silverman, M. 2018, PhRvD, 97, 123008

Quenby, J. 2018, arXiv:1807.01608

Rybicki, G. B., \& Lightman, A. P. 1979, Radiative Processes in Astrophysics (New York: Wiley-Interscience)

Snowden, S. L., Mushotzky, R. F., Kuntz, K. D., \& Davis, D. S. 2008, A\&A, 478,615

Tang, X., \& Piran, T. 2019, MNRAS, 484, 3491

Xi, S.-Q., Liu, R.-Y., Huang, Z.-Q., et al. 2018, arXiv:1810.10928 